SCIENTIFIC REPORT

\title{
Decreased tear lipocalin concentration in patients with meibomian gland dysfunction
}

\author{
M Yamada, H Mochizuki, M Kawai, K Tsubota, T J Bryce
}

Br J Ophthalmol 2005;89:803-805. doi: 10.1136/bjo.2004.055822

Background/aim: Recent studies have demonstrated that tear lipocalin (TL) and phospholipids have a crucial role in maintaining tear film stability. The level of TL in patients with meibomian gland dysfunction (MGD) was examined and these data were correlated with the severity of their clinical disorder.

Methods: 12 patients with obstructive MGD, 12 patients with seborrhoeic MGD, and 12 age matched normal control subjects participated in this study. $3 \mu$ of unstimulated tears were collected with a micropipette from the inferior tear meniscus in the right eye of all subjects. Tear samples were fractionated by high performance liquid chromatography, and TL concentrations were assayed with a bicinchoninic acid technique.

Results: The mean concentration of TL in patients with obstructive and seborrhoeic MGD was significantly lower than in normal controls. TL concentration correlated positively with tear film break up time and negatively with fluorescein staining scores.

Conclusion: These results suggest that TL deficiency may be a predisposing factor for the manifestation of symptoms in MGD.

$\mathrm{T}$ ear lipocalin (TL), formerly called tear specific prealbumin, comprises $15-33 \%$ of the mass of protein in tears. TL is a member of the lipocalin superfamily that is produced in the lacrimal gland as well as in von Ebner's gland..$^{2-4}$ A number of lipophilic substances of different chemical classes, including fatty acids, fatty alcohols, phospholipids, glycolipids and cholesterol, are endogenous ligands of this protein. ${ }^{56}$ Although the physiological roles of TL are not fully understood, recent studies have demonstrated that the low surface tension of tears is the result of a complex of TL with tear lipids. ${ }^{78}$ Glasgow et al have stated that TL may be a key factor in maintaining tear film stability by acting as a lipid scavenger. ${ }^{7}$ This protective function of TL may be compromised in dry eye syndrome. ${ }^{9}$

Meibomian gland dysfunction (MGD), a common, chronic condition of the posterior eyelids, comprises a significant part of dry eye syndrome. ${ }^{10-12}$ The lipid layer, the outermost layer of the tear film produced by the meibomian glands, stabilises tear films by retarding evaporation and lowering surface tension. The compositional changes in lipids of meibum (that is, an increase in polar lipids) in MGD compromise tear film stability, which is clinically manifest as a shortened tear film break up time. ${ }^{12}$

The symptoms of MGD are non-specific and often similar to those of aqueous deficiency dry eye..$^{11}{ }^{12}$ An important clinical feature of MGD is that findings on examination of the lid margin often do not correlate well with the severity of symptoms. ${ }^{12}$ MGD is frequently associated with lacrimal insufficiency, and patients with both conditions are likely to be particularly symptomatic. These observations may suggest the presence of pathogenic factor(s) other than changes in the meibomian glands. In this study, we examined TL concentration in patients with MGD and correlated these results with the severity of their clinical disorder.

\section{SUBJECTS AND METHODS}

Twenty four patients with symptomatic MGD participated in this study. Diagnoses included obstructive MGD in 12 subjects (six female and six male, average age 67.8 (SD 11.5) years, and seborrhoeic MGD in 12 subjects (seven female and five male, age 62.4 (11.3) years). The diagnosis of obstructive MGD was made based on the presence of plugging of the meibomian gland orifices associated with thickened, erythematous eyelid margins. For the assessment of the meibomian gland orifices, digital pressure was applied on the upper tarsus. When cloudy meibum was expressed with more than moderate pressure, or when meibum could not be expressed even with the hard pressure, the diagnosis of obstructive MGD was confirmed. Seborrhoeic MGD was diagnosed based on the excessive expression of meibum, sometime associated with foam along the lateral lower eyelid margin. Twelve age matched normal subjects ( six female and six male, age 61.0 (8.9) years) were used as normal controls.

Most patients had been treated with preservative free artificial tears and/or hyaluronic acid eye drops. Patients using antibiotic eye drops or steroidal eye drops at the time of study, or who had a history of ocular surgery, were excluded. The principles of the World Medical Association Declaration of Helsinki were followed. Each subject received a full explanation of the study, all procedures involved in the study, and provided written informed consent before enrolment. Approval for this investigation was granted by the committee for the protection of human subjects at the Keio University School of Medicine.

Non-masked observers performed a routine ocular examination on all subjects, followed by an examination of the ocular surface, including Schirmer testing, a cotton thread test, vital staining, and measurement of tear film break up time (BUT). For vital staining, $2 \mu \mathrm{l}$ of a saline solution containing $1 \%$ fluorescein and $1 \%$ rose bengal was used. ${ }^{13}$ Results were assessed semiquantitatively using a 0-9 grading scale for fluorescein staining in the cornea and for rose bengal staining in both the cornea and conjunctiva. ${ }^{13}{ }^{14}$ Tear film BUT was measured three times, and the measurements were averaged. Only the right eye of all subjects was used for analysis.

A volume of $3 \mu \mathrm{l}$ of unstimulated tears were collected with a micropipette from the inferior tear meniscus in the right eye of all subjects on a separate day. Samples were stored at $-80^{\circ} \mathrm{C}$ until assay. A volume of $1 \mu \mathrm{l}$ of each tear sample was

Abbreviations: BCA, bicinchoninic acid; BUT, break up time; MGD, meibomian gland dysfunction; TL, tear lipocalin 


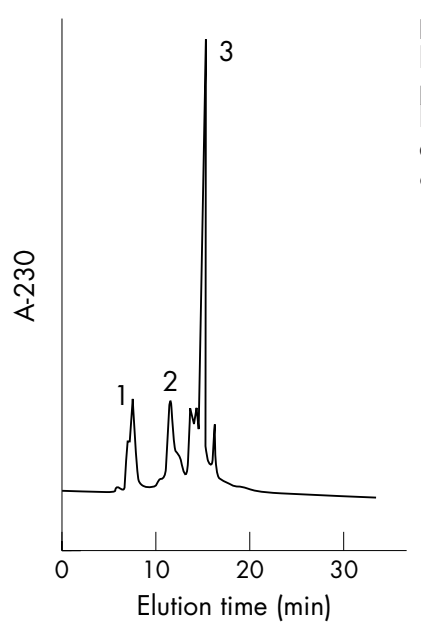

fractionated by high performance liquid chromatography (HPLC). ${ }^{15}$ The determination of peaks in HPLC was carried out by using standards of lactoferrin, albumin, and lysozyme (Sigma, St Louis, MO, USA) based on the report of Baier et al. ${ }^{15}$ The elution was performed using a TSK 3000SWxl column (Tosoh Inc, Tokyo, Japan) with a mobile phase of $0.5 \mathrm{M}$ sodium chloride and $0.1 \mathrm{M}$ sodium phosphate, $\mathrm{pH}$ 5.0. The flow rate was $0.7 \mathrm{ml} / \mathrm{min}$ and detection was performed using a ultraviolet detector set at $230 \mathrm{~nm}$. The fractions containing TL were collected, and then concentrated by evaporation under nitrogen gas. The amount of TL was determined by a bicinchoninic acid (BCA) method. Total protein concentration was also determined by a BCA method using a $1 \mu \mathrm{l}$ tear sample. The final results are expressed as $\mathrm{mg} / \mathrm{ml}$.

\section{RESULTS}

The chromatograms demonstrated excellent separation of TL from other proteins, and were free from interference by endogenous compounds. A typical chromatogram is shown in figure 1 . The retention time of TL was 10.8 minutes.

TL concentration, total protein concentration, and the results of ocular surface examination are shown in table 1.

The mean concentration (SD) of TL in patients with obstructive MGD and seborrhoeic MGD was 0.89 (0.19) mg/ $\mathrm{ml}$, and $1.05(0.22) \mathrm{mg} / \mathrm{ml}$, respectively. In both groups, mean TL concentration was significantly lower than that of normal controls $(p=0.00021$ and $p=0.00042$, respectively, MannWhitney U test). There were no significant differences in total protein concentration among the three groups.

TL concentrations were not significantly correlated with the results of the Schirmer or cotton thread tests, or with the

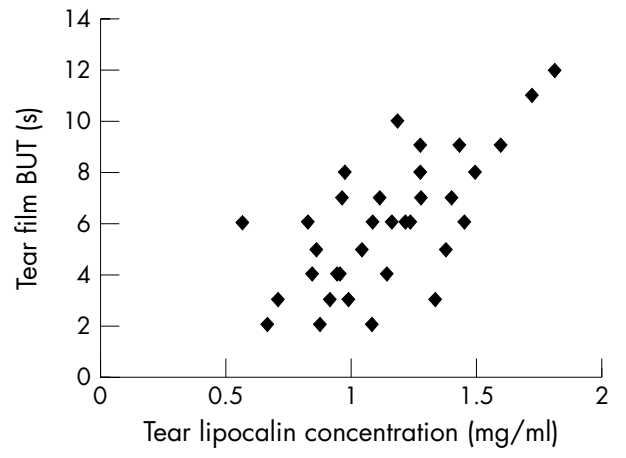

Figure 2 Tear lipocalin concentration was significantly correlated with tear film break up (BUT) time $\left(r=0.67, p=7.8 \times 10^{-6}\right)$.

rose bengal staining score, but did correlate positively with tear film BUT $\left(r=0.67, \mathrm{p}=7.8 \times 10^{-6}\right.$; fig 2$)$ and fluorescein staining scores $\left(r=-0.74, \mathrm{p}=1.8 \times 10^{-7}\right.$; fig 3$)$.

\section{DISCUSSION}

In this study, we have shown that mean TL concentrations in patients with MGD were significantly lower than concentrations in normal controls. This is not likely to be due to decreased tear secretion, because the same volume of tears was collected from all subjects, and the total tear protein concentration did not differ among patients with MGD and normal controls. Regression analyses revealed that TL concentrations were positively correlated with tear film BUT and negatively correlated with fluorescein staining scores. These results suggest that decreased TL concentration is associated with tear film instability and a more severe clinical manifestation of MGD.

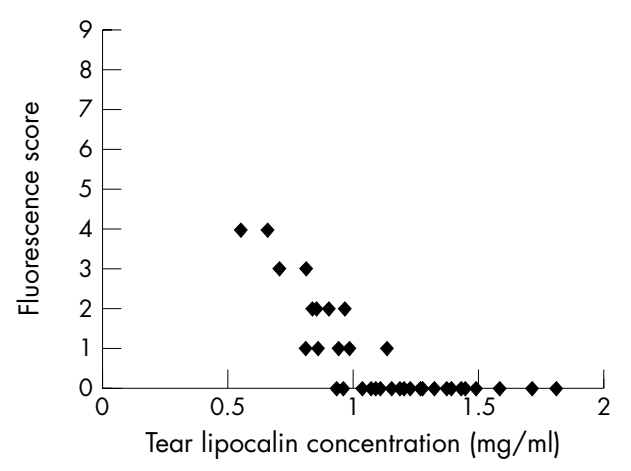

Figure 3 Tear lipocalin concentration was significantly negatively correlated with fluorescence score $\left(r=-0.74, p=1.8 \times 10^{-7}\right)$.

Table 1 Tear lipocalin concentration, total protein concentration, and results of ocular surface examination (SD)

\begin{tabular}{llll}
\hline & Obstructive MGD & Seborrhoeic MGD & Normal controls \\
\hline Schirmer test $(\mathrm{mm})$ & $5.4(2.1)$ & $6.4(2.3)$ & $8.5(3.2)$ \\
Cotton thread test $(\mathrm{mm})$ & $10.4(4.3)$ & $12.7(4.9)$ & $13.7(3.1)$ \\
Tear film BUT $(\mathrm{s})$ & $4.4(1.7)$ & $4.9(2.1)$ & $7.9(1.9)$ \\
Fluorescein score & $1.7(1.5)$ & $0.9(1.1)$ & 0 \\
Rose bengal score & $0.5(0.7)$ & $0.5(0.6)$ & $0.1(0.3)$ \\
Tear lipocalin $(\mathrm{mg} / \mathrm{ml})$ & $0.89(0.19)^{*}$ & $1.05(0.22)^{* *}$ & $1.54(0.23)$ \\
Total protein $(\mathrm{mg} / \mathrm{ml})$ & $8.66(1.50)$ & $9.16(1.76)$ & $9.48(1.72)$ \\
\hline
\end{tabular}

$M C D$, meibomian gland dysfunction.

The concentration of lipocalin in patients with obstructive MGD and seborrhoeic MGD was significantly lower than that of normal controls ( $* p=0.00021$ and ${ }^{* *} p=0.00042$, respectively). 
Recent studies have demonstrated that TL contributes to the high, non-Newtonian viscosity of tear film and its low surface tension..$^{78}$ These actions are thought to be exerted by the lipid binding properties of TL. In the lipocalin family, TL is unique because it binds most strongly the least soluble lipids. $^{56}$ In seborrhoeic MGD, excessive expression of meibum results in excessive concentrations of lipids in tear film. TL may contribute to tear film stability by acting as a lipid scavenger. ${ }^{7}$ In obstructive MGD, low levels of two phospholipids, phosphatidylethanolamine, and sphingomyelin are associated with ocular surface abnormalities. ${ }^{16}$ TL may adjust the lipid composition of tears by binding lipids from the tear fluid and/or releasing lipids into tear fluid. Intensive analysis of lipids compositions of tears and meibum from patients with MGD appears to be essential to test the hypothesis.

Lechner et al have recently reported that the expression of TL in tetracarcinoma derived NT2 precursor cells is enhanced by oxidative stress and that TL binds to several lipid peroxidation products. ${ }^{17}$ These observations suggest that TL acts as a physiological epithelial protective factor by scavenging potentially harmful lipid peroxidation products. TL also has anti-inflammatory and antimicrobial activity. ${ }^{18} 19$ It may exert a regulatory, dampening influence on the inflammatory cascade, thereby protecting against tissue damage from excessive inflammation. The negative correlation between $\mathrm{TL}$ concentration and fluorescein staining scores in the present study may be explained by the protective role of TL.

As mentioned in the introduction, findings on examination of the lid margin often do not correlate well with the severity of symptoms in patients with MGD. ${ }^{12}$ MGD is frequently associated with lacrimal insufficiency, and patients with both conditions are likely to be particularly symptomatic. Our results suggest that decreased TL concentration in tears is one of the pathogenic factors other than changes in the meibomian glands for the development of clinical symptoms in MGD. Further investigations are required to determine the mechanism by which TL exerts protective effects on the ocular surface (that is, stabilising tear film by modulation of tear lipids, scavenging lipid peroxidation products, or regulating excessive inflammation).

To date, however, only a few investigators have reported pathophysiological changes in TL concentrations. The level of TL remains constant in non-stimulated and stimulated tears of normal subjects. ${ }^{20}$ In soft contact lens wearers, the level of lipocalin in tears was significantly higher than that of normal controls. ${ }^{21}$ Intolerant contact lens wearers had significantly higher amounts of lipocalin in their tears than tolerant contact lens wearers. ${ }^{22}$ These observations appear to conflict with the proposed protective role of TL in maintaining tear film stability and epithelial integrity. However, Glasson et al have speculated that the greater concentration of TL observed in tears from intolerant contact lens wearers may be a response to increased lipid peroxidation products. ${ }^{22}$ Further studies should be done to clarify this issue.

In summary, mean TL concentration was significantly lower in patients with MGD than in normal control subjects. TL concentrations correlated positively with tear film BUT and negatively with fluorescein staining scores. Our results suggest that a deficiency of TL may be a predisposing factor for the development of clinical symptoms in MGD.

\section{Authors' affiliations}

M Yamada, H Mochizuki, M Kawai, K Tsubota, Department of Ophthalmology, Keio University School of Medicine, Tokyo, Japan M Yamada, H Mochizuki, Division for Vision Research, National Institute of Sensory Organs, National Tokyo Medical Center, Tokyo, Japan

T J Bryce, Department of Health Sociology, Tokyo University Graduate School of Medicine, Tokyo, Japan

The authors have no proprietary interest in any materials in this manuscript.

Correspondence to: Masakazu Yamada, MD, Division for Vision Research, National Institute of Sensory Organs, National Tokyo Medical Center, 2-5-1 Higashigaoka, Meguro, Tokyo 152-8902, Japan; yamadamasakazu@kankakuki.go.jp

Accepted for publication 6 October 2004

\section{REFERENCES}

1 Fullard RJ, Kissner DM. Purification of the isoforms of tear specific preallbumin. Curr Eye Res 1991;10:613-28.

2 Inada K. Studies of human tear proteins. 3. Distribution of specific tear prealbumin in lacrimal glands and other adnexa. Jpn J Ophthalmol 1984;28:315-30

3 Bläker M, Kock C, Ahlers C, et al. Molecular cloning of human von Ebner's gland protein: a member of the lipocalin superfamily highly expressed in lingual salivary gland. Biochim Biophys Acta 1993;1172:131-7.

4 Redl B, Holzfeind P, Lottspeich F. CDNA cloning and sequencing reveals tear lipocalin to be a member of the lipophilic-ligand carrier protein superfamily. J Biol Chem 1992;267:20282-7.

5 Glasgow BJ, Abduragimov AR, Farahbakhsh ZT, et al. Tear lipocalins bind a broad array of lipid ligands. Curr Eye Res 1995; 14:363-72.

6 Gasymov OK, Abduragimov AR, Yusifov TN, et al. Binding studies of tear lipocalin: the role of the conserved tryptophan in maintaining structure, stability and ligand affinity. Biochim Biophys Acta 1999;1433:307-20.

7 Glasgow BJ, Marshall G, Gasymov OK, et al. Tear lipocalins: potential lipid scavengers for the corneal surface. Invest Ophthalmol Vis Sci 1999;40:3100-7.

8 Nagyová B, Tiffany JM. Components responsible for the surface tension of human tears. Curr Eye Res 1999;19:4-11.

9 Schoenwald RD, Vidvauns S, Wurster DE, et al. Tear film stability of protein extracts from dry eye patients administrated a sigma agonist. $J$ Ocul Pharmacol Ther 1997;13:151-61.

10 Bron AJ, Tiffany JM, Gouveia SM, et al. Functional aspects of the tear film lipid layer. Exp Eye Res 2004;78:347-60.

11 Bron AJ, Tiffany JM. The contribution of meibomian disease to dry eye. Ocular Surface 2004;3:149-64

12 Driver P, Lemp MA. Seborrhea and meibomian gland dysfunction. In: Krachmer JH, Mannis MJ, Holland EJ, eds. Cornea: cornea and external disease: clinical diagnosis and management. St Lovis: Mosby-Year Book, Inc, 1997:625-32.

13 Shimazaki J, Goto E, Ono M, et al. Meibomian gland dysfunction in patients with Siögren syndrome. Ophthalmology 1998;105:1485-8.

14 Van Bijsterveld OP. Diagnostic tests in the sicca syndrome. Arch Ophthalmol 1969:82:10-4.

15 Baier G, Wollensak G, Mur E, et al. Analysis of human tear proteins by differential high-performance liquid chromatographic techniques. J Chromatogr 1990;525:319-28.

16 Shine WE, McCulley JP. Keratoconjunctivitis sicca associated with meibomian secretion polar lipid abnormality. Arch Ophthalmol 1998;116:849-52.

17 Lechner M, Wojnar P, Redl B. Human tear lipocalin acts as an oxidativestress-induced scavenger of potentially harmful lipid peroxidation products in a cell culture system. Biochem J 2001;356:129-35.

18 Gachon A-M F, Lacazette E. Tear lipocalin and the eye's front line of defence. Br J Ophthalmol 1998:82:453-5.

19 Lögdberg L, Wester L. Immunocalins: a lipocalin subfamily that modulates immune and inflammatory responses. Biochim Biophys Acta 2000; 1482:284-97.

20 Fullard RJ, Snyder C. Protein levels in nonstimulated and stimulated tears of normal human subjects. Invest Ophthalmol Vis Sci 1990;31:1119-26.

21 Grus FH, Sabuncuo P, Augustin AJ. Quantitative analyse der trönenproteinmuster bei weichen kontaktlinsen-klinische studie. Klin Monatsbl Augenheilkd 2001;218:239-42.

22 Glasson MJ, Stapleton F, Wilcox MDP. Lipid, lipase and lipocalin differences between tolerant and intolerant contact lens wearers. Curr Eye Res 2002;25:227-35 